Canad. Math. Bull. 22 (3), 1979

\title{
A NOTE ON BERNSTEIN'S BIVARIATE INEQUALITY
}

\author{
BY \\ K. MULLEN
}

\begin{abstract}
SUMMARY. An upper bound for $P\left[\sum X_{i} \geq t \sigma, \sum Y_{i} \geq t \sigma\right]$, where $\left(X_{i}, Y_{i}\right), i=1,2, \ldots, n$ are bounded independent random variables, was given by Mullen (1973). An improvement to the bound is possible without further assumptions.
\end{abstract}

Discussion. If $\left(X_{1}, Y_{1}\right),\left(X_{2}, Y_{2}\right), \ldots,\left(X_{n}, Y_{n}\right)$ are $n$ independent random variables for which $E\left(X_{i}\right)=E\left(Y_{i}\right)=0, \operatorname{Var}\left(X_{i}\right)=\operatorname{Var}\left(Y_{i}\right)=\sigma_{i}^{2}, \operatorname{Cov}\left(X_{i}, Y_{i}\right)=$ $\rho \sigma_{i}^{2}$ and for which $\left|X_{i}\right| \leq R,\left|Y_{i}\right| \leq R$, and if $a, b, t$ are positive real numbers, then Mullen (1973) has shown that

$$
P\left[\sum_{i} X_{i} \geq t \sigma, \sum_{i} Y_{i} \geq t \sigma\right]<\exp \left\{\frac{-t^{2}(2-|\rho|)}{2\left(1+\frac{t R}{3 \sigma}\right)}\right\}
$$

A brief sketch of the proof is as follows. Since

$$
E e^{a X_{i}+b Y_{i}}=1+a b \rho \sigma_{i}^{2}+\frac{a^{2} \sigma_{i}^{2} A_{i}}{2}+\frac{b^{2} \sigma_{i}^{2} B_{i}}{2}+a b \sigma_{i}^{2}\left(C_{i}+D_{i}+E_{i}\right)
$$

where

$$
\begin{gathered}
A_{i}=\sum_{r=2}^{\infty} \frac{a^{r-2} E X_{i}^{r}}{r ! \sigma_{i}^{2} \frac{1}{2}}, \quad B_{i}=\sum_{s=2}^{\infty} \frac{b^{s-2} E Y_{i}^{s}}{s ! \sigma_{i}^{2} \frac{1}{2}} \\
C_{i}=\sum_{r=2}^{\infty} \frac{a^{r-1} E Y_{i} X_{i}^{r}}{r ! \sigma_{i}^{2}}, \quad D_{i}=\sum_{s=2}^{\infty} \frac{b^{s-1} E X_{i} Y_{i}^{s}}{s ! \sigma_{i}^{2}}, \quad E_{i}=\sum_{r=2}^{\infty} \sum_{s=2}^{\infty} \frac{a^{r-1} b^{s-1} E X_{i}^{r} Y_{i}^{s}}{r ! s ! \sigma_{i}^{2}}
\end{gathered}
$$

then

$$
E e^{a X_{i}+b Y_{i}}<\exp \left\{\left(\frac{a^{2} \sigma_{i}^{2}}{2}+\frac{b^{2} \sigma_{i}^{2}}{2}\right) M_{i}+a b \sigma_{i}^{2} G_{i}\right\}
$$

where

$$
M_{i}=\operatorname{Max}\left(A_{i}, B_{i}\right), \quad G_{i}=\rho+C_{i}+D_{i}+E_{i}
$$

and

$$
E e^{a \sum X_{i}+b \sum Y_{i}}<\exp \left\{\left(\frac{a^{2} \sigma^{2}}{2}+\frac{b^{2} \sigma^{2}}{2}\right) M+a b \sigma G\right\}
$$

where

$$
M=\operatorname{Max}_{i} M_{i}, \quad G=\operatorname{Max}_{i} G_{i}, \quad \sigma^{2}=\sum_{i} \sigma_{i}^{2}
$$

Received by the editors April 20, 1978 and, in revised form, July 5, 1978. 
Extending the idea of Chebyshev's inequality, we can write

$$
P\left[\sum X_{i} \geq t \sigma, \sum Y_{i} \geq t \sigma\right] \leq \exp \left\{\left(\frac{a^{2} \sigma^{2}}{2}+\frac{b^{2} \sigma^{2}}{2}\right) M+a b \sigma^{2} G-t \sigma(a+b)\right\}
$$

which is minimized (with respect to $a$ and $b)$ for $M=G=t /(\sigma(a+b))$, so that

$$
P\left[\sum X_{i} \geq t \sigma, \sum Y_{i} \geq t \sigma\right]<\exp \left\{\frac{-t \sigma(a+b)}{2}\right\}
$$

Now if

$$
\left.\begin{array}{l}
E\left|X_{i}\right|^{r} \leq \frac{1}{2} \sigma_{i}^{2} r ! W^{r-2} \\
E\left|Y_{i}\right|^{s} \leq \frac{1}{2} \sigma_{i}^{2} s ! W^{s-2}
\end{array}\right\} \begin{aligned}
& r, s \geq 2 \\
& W \text { a constant }
\end{aligned}
$$

then

$$
A_{i} \leq \frac{1}{1-a w}, \quad B_{i} \leq \frac{1}{1-b W}
$$

and

$$
(\text { for } a>b), \quad M \leq \frac{1}{1-a w}
$$

so that

$$
a \geq \frac{t-\sigma b}{\sigma+t w}
$$

which when substituted into (1) gives

$$
P\left[\sum X_{i} \geq t \sigma, \sum Y_{i} \geq t \sigma\right]<\exp \left\{\frac{-t^{2} \sigma}{2} \frac{(1+b W)}{\sigma+t W}\right\}
$$

Now choosing $b$ so that $1-b W \leq|\rho|$ we have

$$
P\left[\sum X_{i} \geq t \sigma, \sum Y_{i} \geq t \sigma\right]<\exp \left\{\frac{-t^{2}(2-|\rho|)}{2\left(1+t \frac{W}{\sigma}\right)}\right\}
$$

Of particular interest are variables satisfying $\left|X_{i}\right| \leq R,\left|Y_{i}\right| \leq R$, for which

$$
\begin{gathered}
E\left|X_{i}\right|^{r} \leq \sigma_{i}^{2} R^{r-2}, \quad E\left|Y_{i}\right|^{s} \leq \sigma_{i}^{2} R^{s-2}, \quad E\left|Y_{i}\right|\left|X_{i}\right|^{r} \leq|\rho| \sigma_{i}^{2} R^{r-1}, \\
E\left|X_{i}\right|\left|Y_{i}\right|^{s} \leq|\rho| \sigma_{i}^{2} R^{s-1}, \quad E\left|X_{i}\right|^{r}\left|Y_{i}\right|^{s} \leq|\rho| \sigma_{i}^{2} R^{r-1} R^{s-1}
\end{gathered}
$$

so that $R=3 W$ and

$$
P\left[\sum X_{i} \geq t \sigma, \sum Y_{i} \geq t \sigma\right]<\exp \left\{\frac{-t^{2}(2-|\rho|)}{2\left(1+\frac{t R}{3 \sigma}\right)}\right\}
$$


This Bernstein-type inequality, after Bernstein (1924), who developed the univariate case, may be improved upon without further assumptions.

Writing $S(X)$ and $S(Y)$ for $\sum_{i} X_{i}$ and $\sum_{i} Y_{i}$ respectively, then (from Mullen with $a R=x, b R=y, \sigma / R=s$, we have

$$
P[S(X) \geq t \sigma, S(Y) \geq t \sigma]<\exp \left\{\frac{x^{2} s^{2} A}{2}+\frac{y^{2} s^{2} B}{2}+x y s^{2} G-t s(x+y)\right\}
$$

where from the same reference we let $A, B$ and $G$ correspond to $\max _{i} A_{i}$, $\max _{i} B_{i}$ and $\max G_{i}$ respectively, so that

$$
\begin{aligned}
A, B & \leq \frac{9|\rho|}{(3-x)(3-y)} \\
G & \leq \frac{9|\rho|}{(3-x)(3-y)}
\end{aligned}
$$

we may write the exponent of (2) as

$$
\frac{9(x+y)^{2} s^{2}|\rho|}{2(3-x)(3-y)}-t s(x+y)
$$

which is minimized (with respect to $x$ and $y$ ) for

$$
\begin{aligned}
& \frac{9 s|\rho|}{2} \frac{y^{2}-x^{2}+6 x+6 y}{(3-x)^{2}(3-y)}-t=0 \\
& \frac{9 s|\rho|}{2} \frac{x^{2}-y^{2}+6 x+6 y}{(3-x)(3-y)^{2}}-t=0
\end{aligned}
$$

which imply that $x=y$ is the single real zero of

$$
x^{3}-9 x^{2}+x\left(27+\frac{54|\rho| s}{t}\right)-27=0
$$

The results of substituting the roots of (3) into (2) were evaluated for a wide range of $|\rho|, s$ and $t$, and below we give an abbreviated table for $|\rho|=$ $0.2(0.3) 0.8, s=0.5(1) 4.5, t=1(1) 7$. A comparison with Mullen's expression (8) is also given.

The improvement discussed here is analogous to the 'First Improvement' given by Bennett (1962). This leads one to ask if further improvement can be made to the bound, using the ideas of Benuett's "Second Improvement." The author took this approach, but found that for a similar range of $|\rho|, s$ and $t$ that no general improvement could be affected. 
TABLE. Comparing the Old Bound (Mullen's expression (8)) and the Improved Bound (expression (1)).

\begin{tabular}{|c|c|c|c|c|c|c|c|c|c|}
\hline$|\rho|$ & $s$ & $t$ & $\begin{array}{c}\text { Old } \\
\text { Bound }\end{array}$ & $\begin{array}{l}\text { Improved } \\
\text { Bound }\end{array}$ & $|\rho|$ & $s$ & $t$ & $\begin{array}{c}\text { Old } \\
\text { Bound }\end{array}$ & $\begin{array}{c}\text { Improved } \\
\text { Bound }\end{array}$ \\
\hline 0.20 & 0.50 & 0.50 & 0.84472 & 0.75207 & 0.50 & 1.50 & 0.50 & 0.84472 & 0.81241 \\
\hline 0.20 & 0.50 & 1.00 & 0.58275 & 0.44830 & 0.50 & 1.50 & 1.00 & 0.54138 & 0.48510 \\
\hline 0.20 & 0.50 & 1.50 & 0.36331 & 0.24066 & 0.50 & 1.50 & 1.50 & 0.28206 & 0.23350 \\
\hline 0.20 & 0.50 & 2.00 & 0.21377 & 0.12083 & 0.50 & 1.50 & 2.00 & 0.12532 & 0.09528 \\
\hline 0.20 & 0.50 & 2.50 & 0.12131 & 0.05780 & 0.50 & 1.50 & 2.50 & 0.04912 & 0.03401 \\
\hline 0.20 & 0.50 & 3.00 & 0.06721 & 0.02663 & 0.50 & 1.50 & 3.00 & 0.01742 & 0.01086 \\
\hline 0.20 & 0.50 & 3.50 & 0.03661 & 0.01190 & 0.50 & 1.50 & 3.50 & 0.00570 & 0.00315 \\
\hline 0.20 & 1.50 & 0.50 & 0.81669 & 0.65291 & 0.50 & 2.50 & 0.50 & 0.83880 & 0.80087 \\
\hline 0.20 & 1.50 & 1.00 & 0.47885 & 0.25862 & 0.50 & 2.50 & 1.00 & 0.51594 & 0.44664 \\
\hline 0.20 & 1.50 & 1.50 & 0.21899 & 0.07697 & 0.50 & 2.50 & 1.50 & 0.24506 & 0.18839 \\
\hline 0.20 & 1.50 & 2.00 & 0.08272 & 0.01878 & 0.50 & 2.50 & 2.00 & 0.09363 & 0.06333 \\
\hline 0.20 & 1.50 & 2.50 & 0.02689 & 0.00394 & 0.50 & 2.50 & 2.50 & 0.02973 & 0.01759 \\
\hline 0.20 & 1.50 & 3.00 & 0.00775 & 0.00073 & 0.50 & 2.50 & 3.00 & 0.00806 & 0.00415 \\
\hline 0.20 & 1.50 & 3.50 & 0.00203 & 0.00012 & 0.50 & 2.50 & 3.50 & 0.00190 & 0.00085 \\
\hline 0.20 & 2.50 & 0.50 & 0.80982 & 0.61718 & 0.50 & 3.50 & 0.50 & 0.83612 & 0.79525 \\
\hline 0.20 & 2.50 & 1.00 & 0.45198 & 0.19866 & 0.50 & 3.50 & 1.00 & 0.50420 & 0.42732 \\
\hline 0.20 & 2.50 & 1.50 & 0.18498 & 0.04211 & 0.50 & 3.50 & 1.50 & 0.22842 & 0.16682 \\
\hline 0.20 & 2.50 & 2.00 & 0.05830 & 0.00657 & 0.50 & 3.50 & 2.00 & 0.08046 & 0.04975 \\
\hline 0.20 & 2.50 & 2.50 & 0.01472 & 0.00081 & 0.50 & 3.50 & 2.50 & 0.02268 & 0.01176 \\
\hline 0.20 & 2.50 & 3.00 & 0.00307 & 0.00008 & 0.50 & 3.50 & 3.00 & 0.00525 & 0.00226 \\
\hline 0.20 & 2.50 & 3.50 & 0.00054 & 0.00001 & 0.50 & 3.50 & 3.50 & 0.00102 & 0.00036 \\
\hline 0.20 & 3.50 & 0.50 & 0.80672 & 0.59831 & 0.50 & 4.50 & 0.50 & 0.83460 & 0.79192 \\
\hline 0.20 & 3.50 & 1.00 & 0.43967 & 0.16909 & 0.50 & 4.50 & 1.00 & 0.49744 & 0.41566 \\
\hline 0.20 & 3.50 & 1.50 & 0.17001 & 0.02848 & 0.50 & 4.50 & 1.50 & 0.21899 & 0.15416 \\
\hline 0.20 & 3.50 & 2.00 & 0.04861 & 0.00324 & 0.50 & 4.50 & 2.00 & 0.07332 & 0.04235 \\
\hline 0.20 & 3.50 & 2.50 & 0.01064 & 0.00027 & 0.50 & 4.50 & 2.50 & 0.01916 & 0.00893 \\
\hline 0.20 & 3.50 & 3.00 & 0.00184 & 0.00002 & 0.50 & 4.50 & 3.00 & 0.00399 & 0.00149 \\
\hline 0.20 & 3.50 & 3.50 & 0.00026 & 0.00000 & 0.50 & 4.50 & 3.50 & 0.00068 & 0.00020 \\
\hline 0.20 & 4.50 & 0.50 & 0.80496 & 0.58657 & 0.80 & 0.50 & 0.50 & 0.89360 & 0.89153 \\
\hline 0.20 & 4.50 & 1.00 & 0.43260 & 0.15148 & 0.80 & 0.50 & 1.00 & 0.69768 & 0.68681 \\
\hline 0.20 & 4.50 & 1.50 & 0.16162 & 0.02156 & 0.80 & 0.50 & 1.50 & 0.50916 & 0.48394 \\
\hline 0.20 & 4.50 & 2.00 & 0.04348 & 0.00193 & 0.80 & 0.50 & 2.00 & 0.35752 & 0.31991 \\
\hline 0.20 & 4.50 & 2.50 & 0.00869 & 0.00012 & 0.80 & 0.50 & 2.50 & 0.24506 & 0.20128 \\
\hline 0.20 & 4.50 & 3.00 & 0.00132 & 0.00001 & 0.80 & 0.50 & 3.00 & 0.16530 & 0.12167 \\
\hline 0.20 & 4.50 & 3.50 & 0.00016 & 0.00000 & 0.80 & 0.50 & 3.50 & 0.11025 & 0.07112 \\
\hline 0.50 & 0.50 & 0.50 & 0.86882 & 0.85077 & 0.80 & 1.50 & 0.50 & 0.87372 & 0.87095 \\
\hline 0.50 & 0.50 & 1.00 & 0.63763 & 0.60501 & 0.80 & 1.50 & 1.00 & 0.61207 & 0.60650 \\
\hline 0.50 & 0.50 & 1.50 & 0.43009 & 0.38992 & 0.80 & 1.50 & 1.50 & 0.36331 & 0.35583 \\
\hline 0.50 & 0.50 & 2.00 & 0.27645 & 0.23494 & 0.80 & 1.50 & 2.00 & 0.18985 & 0.18173 \\
\hline 0.50 & 0.50 & 2.50 & 0.17242 & 0.13456 & 0.80 & 1.50 & 2.50 & 0.08975 & 0.08263 \\
\hline 0.50 & 0.50 & 3.00 & 0.10540 & 0.07402 & 0.80 & 1.50 & 3.00 & 0.03916 & 0.03400 \\
\hline 0.50 & 0.50 & 3.50 & 0.06353 & 0.03938 & 0.80 & 1.50 & 3.50 & 0.01601 & 0.01282 \\
\hline
\end{tabular}




\begin{tabular}{|c|c|c|c|c|c|c|c|c|c|}
\hline$|\rho|$ & $s$ & $t$ & $\begin{array}{c}\text { Old } \\
\text { Bound }\end{array}$ & $\begin{array}{c}\text { Improved } \\
\text { Bound }\end{array}$ & $|\rho|$ & $s$ & $t$ & $\begin{array}{c}\text { Old } \\
\text { Bound }\end{array}$ & $\begin{array}{c}\text { Improved } \\
\text { Bound }\end{array}$ \\
\hline 0.80 & 2.50 & 0.50 & 0.86882 & 0.86533 & 0.80 & 3.50 & 3.00 & 0.01500 & 0.01384 \\
\hline 0.80 & 2.50 & 1.00 & 0.58895 & 0.58221 & 0.80 & 3.50 & 3.50 & 0.00404 & 0.00360 \\
\hline 0.80 & 2.50 & 1.50 & 0.32465 & 0.31782 & 0.80 & 4.50 & 0.50 & 0.86533 & 0.86116 \\
\hline 0.80 & 2.50 & 2.00 & 0.15036 & 0.14510 & 0.80 & 4.50 & 1.00 & 0.57200 & 0.56326 \\
\hline 0.80 & 2.50 & 2.50 & 0.06005 & 0.05668 & 0.80 & 4.50 & 1.50 & 0.29671 & 0.28836 \\
\hline 0.80 & 2.50 & 3.00 & 0.02113 & 0.01929 & 0.80 & 4.50 & 2.00 & 0.12365 & 0.11838 \\
\hline 0.80 & 2.50 & 3.50 & 0.00666 & 000580 & 0.80 & 4.50 & 2.50 & 0.04225 & 0.03975 \\
\hline 0.80 & 3.50 & 0.50 & 0.86660 & 0.86269 & $\begin{array}{l}0.80 \\
0.80\end{array}$ & $\begin{array}{l}4.50 \\
4.50\end{array}$ & $\begin{array}{l}3.00 \\
3.50\end{array}$ & $\begin{array}{l}0.01206 \\
000292\end{array}$ & 0.01110 \\
\hline 0.80 & 3.50 & 1.00 & 0.57821 & 0.57033 & & & & & \\
\hline 0.80 & 3.50 & 1.50 & 0.30689 & 0.29933 & & & & & \\
\hline 0.80 & 3.50 & 2.00 & 0.13319 & 0.12815 & & & & & \\
\hline 0.80 & 3.50 & 2.50 & 0.04837 & 0.04572 & & & & & \\
\hline
\end{tabular}

\section{REFERENCES}

1. G. Bennett, (1962), Probability Inequalities for the Sum of Independent Random Variables. J. Amer. Statist. Ass. 57, 33-45.

2. S. Bernstein, (1924), Sur une modification de l'inégalité de Tchebichef (In Russian, French Summary). Ann. Sci. Inst. Sew. Ukraine Sect. Math I.

3. K. Mullen, (1973), Bernstein's Inequality in the Bivariate Case. Can. Math. Bull 16, 83-86.

DEPARTMENT OF MATHEMATICS

UNIVERSITY OF GUELPH

GuelPh, ONT. NIG 2W1 